\title{
Transport of light in amorphous photonic materials
}

\author{
Mathias Reufer \\ Department of Physics, University of Fribourg, 1700 Fribourg, Switzerland
}

Luis Fernando Rojas-Ochoa

Departamento de Física, Cinvestav-IPN, Av. Instituto Politécnico Nacional 2508, 07360 México Distrito

Federal, Mexico

Stefanie Eiden

Physics Department, University of Konstanz, D-78457 Konstanz, Germany

\section{Juan José Sáenz}

Departamento de Física de la Materia Condensada and Instituto "Nicolás Cabrera," Universidad Autónoma de Madrid, E-28049 Madrid, Spain

Frank Scheffold ${ }^{\text {a) }}$

Department of Physics and Fribourg Center for Nanomaterials, University of Fribourg, 1700 Fribourg, Switzerland

\begin{abstract}
Amorphous photonic materials based on dense assemblies of high refractive index spherical particles are presented. Light transmission through these photonic glasses shows a nontrivial wavelength dependence. The transmission spectra can be quantitatively reproduced by modeling the optical properties starting from their building blocks. Our results emphasize the relevance of including short range order correlations and an appropriate effective refractive index in the analysis of light transmission through amorphous photonic materials.
\end{abstract}

The propagation of light in a dense dielectric depends on refractive index variations and structural order. Perfectly ordered photonic crystals provide a set of unique options to control the electromagnetic states and light transport without radiation and absorption losses. ${ }^{1-5}$ Significant effects are usually obtained for strongly photonic materials, i.e., those with a refractive index ratio or scattering contrast $n_{\text {particle }} / n_{\text {medium }}$ of 2-3 or larger. In disordered dielectrics, in the absence of long range order, light is spread out diffusively. However, strong scattering of light in amorphous dielectrics can also lead to unusual photonic behavior ${ }^{6-8}$ often related to precursors of Anderson localization of light such as coherent backscattering or mesoscopic fluctuations. ${ }^{9,10}$ Understanding the optical properties of amorphous photonic materials is not only a problem of fundamental interest but also a key issue in the development of noninvasive imaging tools and optical materials. Despite this importance there is still a lack of information on how to predictably tailor photonic amorphous systems starting from their building blocks.

Outside the localized regime $\left(k l^{*}>1\right)$ the transport of light in a disordered dielectric can be described as a diffusion process with a transport mean free path $l^{*}$. The dimensionless parameter $k l^{*}$ is a measure of the scattering strength for a wave number $k$. In order to design photonic materials with very small values of $k l^{*}$, both the structural properties and the scattering cross section have to be optimized. The latter requires scattering objects to have a high refractive index contrast. Unfortunately there is practically no experimental data available on dense assemblies of high index particles with well-defined shape. In this letter, we report on the

\footnotetext{
${ }^{\text {a) }}$ Author to whom correspondence should be addressed. Electronic mail: frank.scheffold@unifr.ch
}

preparation of assemblies of high refractive index spheres and we present a quantitative approach to model the transmission spectra.

A quantitative relation between $l^{*}$ and the microscopic scattering properties is given by the number density $\rho$ and the transport cross section $\sigma_{\mathrm{tr}}, 11,12$

$$
\frac{1}{l^{*}}=\rho \sigma_{\mathrm{tr}}=\frac{\pi}{k^{6}} \int_{0}^{2 k} \rho F(q) S(q) q^{3} d q,
$$

where $q=2 k \sin (\theta / 2)$ for a scattering angle in the range of $\theta=0-180^{\circ}$. This is the same relation used to describe the electrical conductance of liquid and amorphous metals. ${ }^{13,14}$ The optical form factor $F(q)$ is given by the differential cross section of an isolated particle. For uncorrelated scatterers (dilute limit) the optical density $1 / l^{*}$ increases linearly with the particle volume fraction $1 / l^{*} \propto \Phi$. As the concentration increases, particle interactions induce positional correlations which have a substantial influence on the $l^{*}$ values. ${ }^{12}$ For systems with weak scattering contrast, the validity of Eq. (1) has been confirmed experimentally for different particle sizes and mixtures. $12,15,16$

It was frequently argued that optimal random photonic materials cannot be designed using spherical objects (since structural order can lead to an increase of $l^{*}$ ) and as a consequence, most previous studies used randomly shaped and highly polydisperse systems ${ }^{17}$ or porous structures such as electrochemically etched gallium phosphide. ${ }^{6}$ Modeling the optical properties of such systems starting from the individual blocks was then a formidable task. However, it has recently been pointed out by Rojas-Ochoa et al. that while the packing of spheres induces an increase in $l^{*}$ in the longwavelength $k d>\pi$ limit, close to the Bragg condition, structural order can actually lead to a reduced value of $l^{*}$ as com- 


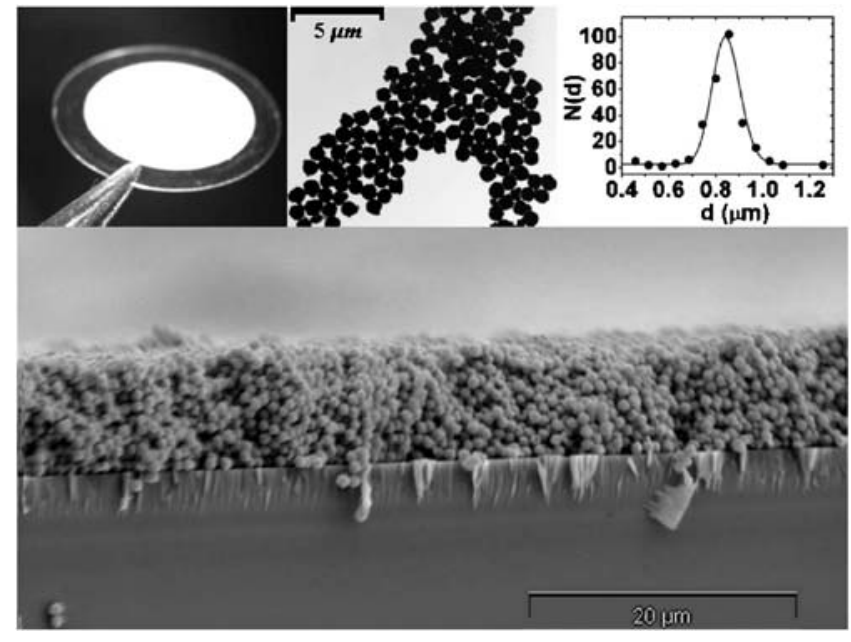

FIG. 1. Characterization of $\mathrm{TiO}_{2}$ particles. Upper panels from left to right: (a) Thin colloidal film on a glass substrate (diameter of $15 \mathrm{~mm}$ ), (b) transmission electron microscopy picture of individual particles, and (c) particle sizes fitted with a Schultz distribution reveals a mean diameter of $845 \mathrm{~nm}$ with a polydispersity of 7\%. Main panel: Scanning electron microscopy side view of an amorphous thin film of $\mathrm{TiO}_{2}$ particles, with thickness of $L$ $=8.8 \mu \mathrm{m}$

pared to an uncorrelated system. ${ }^{18}$ Recent work on the effect of structural correlations on optical properties has renewed the interest in this problem. ${ }^{18-21}$ As we will show, it is possible to design photonic materials based on dense assemblies of high refractive index, strongly correlated spherical particles for which the scattering properties can be determined accurately.

As primary particles we have synthesized spherical and rather monodisperse titanium dioxide particles following the approach reported in Ref. 22. Sintering at $1000{ }^{\circ} \mathrm{C}$ leads to compact particles made of bulk $\mathrm{TiO}_{2}$ with a rutile crystalline phase, as described in Ref. 23. We further note that subsequent thermal treatment of the final colloidal thin films up to $1050{ }^{\circ} \mathrm{C}$ did not lead to any measurable change of the optical properties. From transmission electron microscopy we find a mean particle diameter $d=845 \mathrm{~nm}$ and a polydispersity of $7 \%$ (Fig. 1). Rutile $\mathrm{TiO}_{2}$ is birefringent, with different refractive indices for light polarized perpendicular $\left(n_{0}\right)$ and parallel $\left(n_{e}\right)$ to the optic axis. Here, we use the weighted sum approximation to determine the mean refractive index $n_{\text {particle }}$ of the particles, as described in Ref. 24. The values for bulk $\mathrm{TiO}_{2}$ are taken from the literature ${ }^{25}$ with $n$ approximately 2.5 in the infrared region probed. By rapid centrifugation on a glass slide (commercial round microscopy cover plates, with diameter of $15 \mathrm{~mm}$ and a thickness of $0.15 \mathrm{~mm}$ ) we prepare dense sediments with thicknesses ranging from 8 to $90 \mu \mathrm{m}$. The particles are dispersed in ethanol and a volume of $800 \mu \mathrm{l}$ is sedimented in a centrifuge at a speed corresponding to $3800 \mathrm{G}$. After removing the supernatant the sediment is dried in the centrifuge at the same speed at $40{ }^{\circ} \mathrm{C}$. The particles are randomly packed due to the forced sedimentation, as shown by the scanning electron microscopy image in Fig. 1 . We estimate the particle density to be $\Phi \simeq 0.65$, near to a maximally random jammed state. The sediments are found to be crack-free over the whole area of approximately $9 \mathrm{~mm}$. We record the wavelength dependent transmission with a commercial ultraviolet-visible (VIS)-near infrared (NIR) spectrometer equipped with an integrating sphere (Fig. 2).

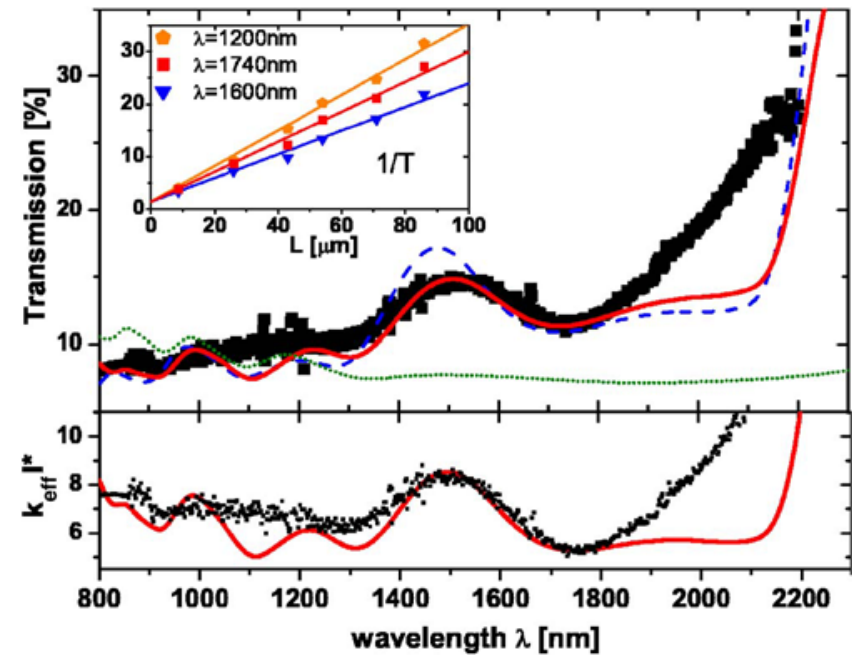

FIG. 2. (Color online) Optical transmission through a sediment with thickness $L=26 \mu \mathrm{m}$ of $845 \mathrm{~nm} \mathrm{TiO}_{2}$ particles. Upper panel: The transmission shows a minimum at $1750 \mathrm{~nm}$. Theoretical lines are calculated from Eqs. (1) and (2) with $\alpha=0.73$ and a volume filling fraction of $\Phi \simeq 65 \%$. Solid line: Percus-Yevick structure factor and a polydispersity of $7 \%$. Dashed line: Prediction for a corresponding monodisperse system. Dotted line: Prediction in the absence of positional correlations $[S(q) \equiv 1$, polydispersity of $7 \%]$. Inset: Inverse of the experimental transmission coefficient as a function of film thickness. The lower panel shows the corresponding $k_{\mathrm{eff}} l^{*}$ values.

In the limit of nonabsorbing and thick slabs (with thickness $\left.L \gtrsim 5 l^{*}\right)$, the transmission coefficient $T$ is given by

$$
T=\left(l^{*}+z_{e}\right) /\left(L+2 z_{e}\right),
$$

where the extrapolation length $z_{e}$ can be calculated by the angle-dependent and polarization averaged reflectivity of the slab/exterior boundary for a given sample refractive index. ${ }^{11}$ The inset of Fig. 2 demonstrates that our system obeys the linear scaling of $1 / T$ as a function of the film thickness $L$ as predicted by Eq. (2). The agreement further confirms that our films are homogenous and crack-free up to almost $100 \mu \mathrm{m}$.

In order to compare the results with Eq. (2), we have to make an assumption about the effective index $n_{\text {eff }}$ (with $k_{\text {eff }}=2 \pi n_{\text {eff }} / \lambda$, where $\lambda$ denotes the wavelength in vacuum) since on average, the scattering contrast will decrease with increasing volume filling. Frequently, the socalled Maxwell-Garnett theory (MGT) is used to estimate $n_{\text {eff }}$ despite the fact that Maxwell-Garnett theory is only valid in the long-wavelength limit $(\lambda \gg d) .{ }^{26}$ Rather than referring to MGT, our description of the $n_{\text {medium }}=n_{\text {eff }}$ is based on an interpolation scheme. For convenience we use the MGT prediction as a starting point and introduce a correction parameter $\alpha$ such that

$$
n_{\mathrm{eff}}(\lambda)=\alpha\left[n_{\mathrm{MGT}}(\lambda)-1\right]+1 .
$$

In general, this correction factor is wavelength dependent and has to approach one in the MGT limit.

For a quantitative analysis we calculate the particle form factor from Mie theory ${ }^{27}$ and the structure factor $S(q)$ by solving the Ornstein-Zernike equation through the PercusYevick closure relation. In the calculation for polydisperse systems, we use a three-component histogram of sizes to represent the polydisperse form and structure factors, which provides a good approximation for the local differential cross section. We find a good agreement between the experimental data and our theoretical calculations over the whole range of accessible wavelengths for $\alpha=0.73 \pm 0.01$. If one takes into 
account the limited accuracy in determining the particle size from transmission electron microscopy, the actual error should be of the order of \pm 0.05 . Our experimental value of $\alpha$ is in reasonable agreement with Brewster angle measurements of Rivas et al. ${ }^{28}$ that indicate $\alpha \approx 0.66$. We find a minmum value for $k_{\mathrm{eff}}{ }^{*}=5.25 \pm 0.12$ at $\lambda=1740 \mathrm{~nm}$ (inset of Fig. 2). It is worthwhile to point out that Eqs. (1) and (2) both describe the absolute values of $T$ and the spectral features such as the minima and maxima in $T(\lambda)$. Significant deviations only appear around $\lambda=2000 \mathrm{~nm}$. This regime is governed by interferences from the shell of first neighbors which are responsible for the first minima in $T(\lambda)$. We think that the slightly nonspherical shape and imperfect packing are responsible for these deviations.

Finally, we would like to point out that for a given value of the experimental transmission coefficient $T$, the value of $n_{\text {eff }}$ significantly impacts the value of $l^{*}$ obtained from Eq. (2). The reason is that the surface reflectivity and thus the extrapolation length $z_{e}$ strongly depend on $n_{\text {eff. }}$ Using the common Maxwell-Garnet refractive index would then lead to an overestimation of the extrapolation length. As a consequence, $l^{*}$ values extracted from Eq. (2) using an experimental transmission coefficient would appear artificially low. Similar arguments hold for the analysis of the coherent backscattering cone often used as an alternative approach to determine $l^{*}$. Therefore, some of the reported $l^{*}$ could be 20\%-30\% lower than the actual values. For example, in our case, we obtain $l^{*}=0.92 \mu \mathrm{m}$ for $n_{\mathrm{eff}}=1.58\left(z_{e}=2.82 l^{*}\right)$ at $\lambda=1740 \mathrm{~nm}$, close to the most pronounced minimum. Based on the Maxwell-Garnet refractive index $n_{\text {eff }}=1.79$ $\left(z_{e}=4.0 l^{*}\right)$ we obtain $l^{*}=0.7 \mu \mathrm{m}$.

Another theoretical approach to determine $l^{*}$ for electromagnetic waves is based on different extensions of the coherent potential approximation (CPA) (for acoustic waves, see Ref. 29). For example, the energy-density CPA introduced by Busch and Soukoulis determines self-consistently the effective refractive index and can be applied to high index materials. ${ }^{30}$ However, in its current implementation, structural correlations are taken into account qualitatively by using independent scatterers with an effective coating. We believe that an extension of the model taking into account the detailed shape of the structure factor of the amorphous photonic medium might open a way to model the experimental data without any adjustable parameters.

In summary, we have shown that it is possible to design strongly photonic materials, based on dense assemblies of high refractive index spherical particles, for which the scattering properties can be determined accurately.

We thank S. Albaladejo, L. Froufe-Pérez, and P. Schurtenberger for interesting discussions, Samiul Amin and
Nasser Ben Braham for help in the early stages of the project, Johanna S. Schütz-Widoniak for help with the particle synthesis, Daniel Baumann for help with the VIS-NIR experiments, and Jean-Claude Bünzli for giving us access to their VIS-NIR spectrometer. This work was partially supported by the Spanish FIS2006-11170-C02-02, the EU-IP "Molecular Imaging" (No. LSHG-CT-2003-503259), the Swiss National Science Foundation (Project No. 200020109137), and KTI/Top Nano 21 (Project No. 5971.2 TNS-NM).

${ }^{1}$ E. Yablonovitch, Phys. Rev. Lett. 58, 2059 (1987).

${ }^{2}$ S. John, Phys. Rev. Lett. 58, 2486 (1987).

${ }^{3}$ K. M. Ho, C. T. Chan, and C. M. Soukoulis, Phys. Rev. Lett. 65, 3152 (1990).

${ }^{4}$ Photonic Crystals and Light Localization in the 21st Century, C. M. Soukoulis, NATO Advanced Studies Institute Series C: Mathematical and Physics Sciences (Kluwer, Dordrecht, 2001), Vol. 563, p. 25.

${ }^{5}$ J. D. Joannopoulos, R. D. Meade, and J. N. Winn, Photonic Crystals: Molding the Flow of Light (Princeton University Press, Princeton, NJ, 1995), p. 8.

${ }^{6}$ F. J. P. Schuurmans, D. Vanmaekelbergh, J. van de Lagemaat, and A. Lagendijk, Science 284, 141 (1999).

${ }^{7}$ C. M. Aegerter, M. Storzer, and G. Maret, Europhys. Lett. 75, 562 (2006).

${ }^{8}$ P. Vukusic, B. Hallam, and J. Noyes, Science 315, 348 (2007).

${ }^{9}$ S. John, Phys. Today 44(5), 32 (1991).

${ }^{10}$ F. Scheffold and G. Maret, Phys. Rev. Lett. 81, 5800 (1998).

${ }^{11}$ J. X. Zhu, D. J. Pine, and D. A. Weitz, Phys. Rev. A 44, 3948 (1991).

${ }^{12}$ S. Fraden, and G. Maret, Phys. Rev. Lett. 65, 512 (1990).

${ }^{13}$ N. W. Ashcroft, and J. Lekner, Phys. Rev. 145, 83 (1966).

${ }^{14}$ P. Haussler, Phys. Rep. 222, 65 (1992).

${ }^{15}$ P. D. Kaplan, A. G. Yodh, and D. J. Pine, Phys. Rev. Lett. 68, 393 (1992).

${ }^{16}$ L. F. Rojas-Ochoa, S. Romer, F. Scheffold, and P. Schurtenberger, Phys. Rev. E 65, 051403 (2002).

${ }^{17}$ J. G. Rivas, R. Sprik, C. M. Soukoulis, K. Busch, and A. Lagendijk, Europhys. Lett. 48, 22 (1999).

${ }^{18}$ L. F. Rojas-Ochoa, J. M. Mendez-Alcaraz, J. J. Senz, P. Schurtenberger, and F. Scheffold, Phys. Rev. Lett. 93, 073903 (2004).

${ }^{19}$ D. Nau, A. Schonhardt, C. Bauer, A. Christ, T. Zentgraf, J. Kuhl, M. W. Klein, and H. Giessen, Phys. Rev. Lett. 98, 133902 (2007).

${ }^{20}$ F. Caton, C. Baravian, and J. Mougel, Opt. Express 15, 2847 (2007).

${ }^{21}$ A. Derode, V. Mamou, and A. Tourin, Phys. Rev. E 74, 036606 (2006).

${ }^{22}$ S. Eiden-Assmann, J. Widoniak, and G. Maret, Chem. Mater. 16, 6 (2004).

${ }^{23}$ J. S. Schütz-Widoniak, "Synthesis and characterization of colloidal inorganic amd metallic particles for optical and other applications," Ph.D. thesis, University of Konstanz, 2005.

${ }^{24}$ E. S. Thiele and R. H. French, Adv. Mater. (Weinheim, Ger.) 10, 1271 (1998).

${ }^{25}$ M. W. Ribarsky, Handbook of Optical Constants of Solids, edited by E. D. Palik (Academic, Orlando, 1985), p. 795.

${ }^{26}$ P. Mallet, C. A. Guérin, and A. Sentenac, Phys. Rev. B 72, 014205 (2005).

${ }^{27}$ M. Kerker, The Scattering of Light and Other Electromagnetic Radiation (Academic, New York, 1959), p. 97.

${ }^{28}$ J. G. Rivas, D. H. Dau, A. Imhof, R. Sprik, B. P. J. Bret, P. M. Johnson, T. W. Hijmans, and A. Lagendijk, Opt. Commun. 220, 17 (2003).

${ }^{29}$ H. P. Schriemer, M. L. Cowan, J. H. Page, P. Sheng, Z. Y. Liu, and D. A. Weitz, Phys. Rev. Lett. 79, 3166 (1997).

${ }^{30}$ K. Busch and C. M. Soukoulis, Phys. Rev. B 54, 893 (1996). 